\section{Band 16, Heft 4, August 2009}

Editorial

216 Komplementärmedizin in der Verfassung: Die Schweizer Bevölkerung hat entschieden Saller, R. (Zürich)

Originalarbeiten

217 Wirksamkeit und Sicherheit von Mistelzubereitungen (Viscum album) für Patienten mit Krebserkrankungen. Ein systematisches Review Melzer, J.; Iten, F.; Hostanska, K.; Saller, R. (Zürich)

227 Homöopathische Behandlung von chronischem Kopfschmerz (ICD-9: 784.0) - eine prospektive Beobachtungsstudie mit 2-Jahres-Follow-Up Witt, C.M. (Berlin); Lüdtke, R. (Essen); Willich, S.N. (Berlin)

237 Reduktion der arteriosklerotischen Nanoplaquebildung und -größe durch $\omega$-3-Fettsäuren bei Patienten nach Herzklappenoperation

Koppe, C.; Rodríguez, M. (Berlin); Winkler, K. (Freiburg i.Br.); Pietzsch, J. (Dresden); Neumann, K.; Hiemann, N.E.; Hetzer, R. (Berlin); Malmsten, M. (Uppsala); Siegel, G. (Berlin/Uppsala)

246 Schmerzlindernde Wirkung von Canthariden-pflaster bei lumbaler Spinalkanalstenose

Rampp, T.; Michalsen, A. (Duisburg/Essen); Lüdtke, R. (Essen); Musial, F. (Duisburg/Essen); Kremer, G. (Duisburg);

Dobos, G.J. (Duisburg/Essen)

Kurzmitteilung

251 Komplementärmedizin in Schweizer Praxen der Grundversorgung

Déglon-Fischer, A.; Barth, J.; Ausfeld-Hafter B. (Bern)

\section{Essay}

256 Unter Druck: Die Homöopathie in Großbritannien und ihre Gegner

Milgrom, L.R. (London)
Vol. 16, Issue 4, August 2009

Editorial

216 Complementary Medicine in the Federal Constitution: The Swiss Vote Saller, R. (Zürich)

Original Articles

217 Efficacy and Safety of Mistletoe Preparations (Viscum album) for Patients with Cancer Diseases. A Systematic Review Melzer, J.; Iten, F.; Hostanska, K.; Saller, R. (Zürich)

227 Homeopathic Treatment of Chronic Headache (ICD-9: 784.0) - a Prospective Observational Study with 2-Year Follow-Up

Witt, C.M. (Berlin); Lüdtke, R. (Essen); Willich, S.N. (Berlin)

237 Reduction of Arteriosclerotic Nanoplaque Formation and Size by n-3 Fatty Acids in Patients after Valvular Defect Operation

Koppe, C.; Rodríguez, M. (Berlin); Winkler, K. (Freiburg i.Br.); Pietzsch, J. (Dresden); Neumann, K.; Hiemann, N.E.; Hetzer, R. (Berlin); Malmsten, M. (Uppsala); Siegel, G. (Berlin/Uppsala)

246 Pain-Relieving Effect of Cantharidin Blister on Lumbar Spinal Stenosis

Rampp, T.; Michalsen, A. (Duisburg/Essen); Lüdtke, R. (Essen); Musial, F. (Duisburg/Essen); Kremer, G. (Duisburg); Dobos, G.J. (Duisburg/Essen)

Short Communication

251 Complementary and Alternative Medicine in Primary Care in Switzerland

Déglon-Fischer, A.; Barth, J.; Ausfeld-Hafter B. (Bern)

Essay

256 Under Pressure: Homeopathy UK and Its Distractors Milgrom, L.R. (London)

\section{KARGER}

Fax +497614520714

Information@Karger.de

www.karger.com 


\section{Band 16, Heft 3, Juni 2009}

Proceedings

262 10. Symposium zur Ratio und Plausibilität in der Naturheilkunde: Ernährung als Chance zur Gesunderhaltung und Wiedergutmachung Gast-Herausgeber: Uhlemann, C. (Jena/Bad Wörishofen)

Journal Club

271 Effekte verschwinden im Genomnebel

273 Was fehlt in der Women's Health Initiative Studie über Multivitamine und Krebs oder kardiovaskuläre Risiken? - Nutrigenomics!

274 Keine Rückschlüsse aus der Unwirksamkeit von Ginkgo für Prävention auf Behandlung, bitte

276 Lassen Sie sich berühren

Abstract Service

277 Latest Publications You Should Not Miss

Gesellschaftsmitteilungen

279 Schweizerische Medizinische Gesellschaft für Phytotherapie (SMGP)

281 Buchbesprechungen

283 News / Ticker

285 Fort- und Weiterbildung

286 Tagungen und Kongresse

287 Hinweise für Autoren

250 Impressum

Einen Ausblick auf den Inhalt der kommenden Hefte finden Sie auf Seite 288.
Vol. 16, Issue 3, June 2009

Proceedings

262 10th Symposium on Reason and Plausibility in Natural Medicine: Nutrition - a Chance for Health Maintenance and Compensation Gast-Herausgeber: Uhlemann, C. (Jena/Bad Wörishofen)

Journal Club

271 Effects Vanish in the Fog of Multigene Analysis

273 What Is Missing in the WHI Report on Multivitamins and Cancer or Cardiovascular Risks? - Nutrigenomics!

274 No Conclusions of Efficacy of Ginkgo for the Treatment of Dementia Possible from a Prevention Trial 276 Be Touched

Abstract Service

277 Latest Publications You Should Not Miss

Society Bulletins

279 Schweizerische Medizinische Gesellschaft für Phytotherapie (SMGP)
281 Book Reviews
283 News / Ticker
285 Education
286 Meetings and Conferences
287 Guidelines for Authors
250 Imprint 